\title{
Extraction of Underwater Acoustic Weak Signal Based on Volterra Filter
}

\author{
Meng Qingxin ${ }^{1,2}$, Yang Shie ${ }^{1,2, a}$, Piao Shengchun ${ }^{1,2}$, Huang Yiwang ${ }^{1,2}$ \\ ${ }^{1}$ Acoustic Science and Technology Laboratory, Harbin Engineering University, Harbin 150001, \\ China; \\ ${ }^{2}$ College of Underwater Acoustic Engineering, Harbin Engineering University, Harbin, 150001, \\ China; \\ ayangshie@hrbeu.edu.cn
}

Keywords: signal extraction; Volterra filter; one step prediction; underwater acoustics

\begin{abstract}
A new method based on Volterra filter is introduced to extract weak signal in strong chaotic noise. A nonlinear prediction model is established by combining phase space reconstruction and adaptive Volterra filter. Self-noise of ship is used for training the prediction model. The model has a good prediction performance of noise without signal, but when signal is mixed with noise, the modal mismatches and prediction error varies dramatically. The cross-correlation of extracted signal and real signal is calculated, and the similarity of waveforms is compared. It had been shown that the algorithm is powerful for regular signal extraction.
\end{abstract}

\section{Introduction}

Conventional signal extraction techniques are based on statistical theory, which is a significant part in signal processing. But in condition of low signal-to-noise ratio (SNR), performance of conventional method degrades. Recently, chaotic theory has been developing rapidly. Chaotic phenomena had been found in ship-radiated noise ${ }^{[1,2]}$, marine ambient noise ${ }^{[3]}$ and ocean reverberation ${ }^{[4]}$. Corresponding research results are widely applied to underwater acoustic signal processing ${ }^{[5,6]}$. Nonlinear dynamical system can be modeled by some methods such as artificial neural network (ANN) ${ }^{[7]}$, Volterra filter ${ }^{[8]}$, Support Vector Machine (SVM) ${ }^{[9]}$ and so on. Because output of Volterra filter is a linear function of the kernel and performance of Volterra filter can be analyzed by linear techniques, Volterra filter is used extensively as a prediction model. In this paper, a method of weak signal extraction from chaotic noise is introduced. The prediction model of chaotic time series is established by applying Volterra filter. The model has a good prediction performance of chaotic noise. When regular signal is mixed with chaotic noise, the model mismatches and prediction error varies dramatically. In underwater acoustic detection, the target is a certain ship-radiated noise, and interferences include ship-radiated noise, ambient noise, and so on. We apply the model above to detect target signal and ship noise, verifying the validity of the algorithm.

\section{Theory and Method}

\section{Modeling of Chaotic Time Series}

Chaos is deterministic, namely, chaotic time series obey certain laws. In fact, chaotic time series can be predicted in a short term, and may occupy preferable prediction performance ${ }^{[10]}$.This provides foundation for modeling chaotic time series. According to coordinate delay method ${ }^{[9]}$, time series $\{x(n), n=1,2, \ldots, N\}$ can be reconstructed, and the point of phase space is:

$$
X(n)=[x(n), x(n-\tau), \cdots, x(n-(m-1) \tau)]^{T}
$$

where $m$ is embedding dimension, and $\tau$ is delay time. It is proved by Takens theorem that if embedding dimension $m \geq 2 d+1$ ( $d$ is the fractal dimension of the attractor), the reconstructed dynamic system is equivalent to the original one in topology, that is, the two strange attractors are homeomorphic in differentiation. Hence, there is a smooth mapping $f: R^{m} \rightarrow R^{m}$, which tracks points in phase space 
where $f(\bullet)$ is the prediction model.

$$
X(n+1)=f(X(n))
$$

\section{Volterra Filter}

The output $y(n)$ of Volterra filter is expressed in the form of Volterra series expansion:

$$
\begin{gathered}
y(n)=h_{0}+\sum_{k=1}^{p} y_{k}(n) \\
y_{k}(n)=\sum_{i_{1}, \cdots, i_{k}=0}^{m-1} h_{k}\left(i_{1}, \cdots, i_{k}\right) \prod_{j=1}^{k} x\left(n-i_{j} \tau\right)
\end{gathered}
$$

where $h_{k}\left(i_{1}, \cdots, i_{k}\right)$ is the coefficient of Volterra filter, namely Volterra kernel, and $p$ represents the order of the filter. In practice, $p$ is usually 2 , and $y(n)$ can be written as:

$$
y(n)=h_{0}+\sum_{i_{1}=0}^{m-1} h_{1}\left(i_{1}\right) x\left(n-i_{1} \tau\right)+\sum_{i_{1}, i_{2}=0}^{m-1} h_{2}\left(i_{1}, i_{2}\right) x\left(n-i_{1} \tau\right) x\left(n-i_{2} \tau\right)
$$

The equation above indicates Volterra filter is a nonlinear adaptive FIR filter, whose coefficient vector and input vector can be also expressed as

$$
\begin{gathered}
H(n)=\left[h_{0}, h_{1}(0), h_{1}(1), \cdots, h_{1}(m-1), h_{2}(0,0), h_{2}(0,1), \cdots, h_{2}(m-1, m-1)\right]^{T} \\
U(n)=\left[1, x(n), x(n-\tau), \cdots, x(n-(m-1) \tau), x^{2}(n), x(n) x(n-\tau), \cdots, x^{2}(n-(m-1) \tau)\right]^{T}
\end{gathered}
$$

So Eq.6 can be written as follow

$$
y(n)=H^{T}(n) U(n)
$$

where the input vector $U(n)$ is obtained by phase space reconstruction, and the coefficient vector $H(n)$ is gained by training Volterra series kernel. The output of Volterra filter is actually a fitting result, realizing time series prediction.

\section{Extraction of Weak Signal}

Suppose the signal $s(n)$ is mixed with chaotic noise $c(n)$, then the observation is $x(n)=s(n)+c(n)$. The forecasted result is $\hat{x}(n)$ and the prediction error is $e(n)=x(n)-\hat{x}(n) e(n)$ can be also expressed as :

$$
e(n)=x(n)-\hat{x}(n)=s(n)+c(n)-\hat{x}(n)
$$

If the accuracy of nonlinear prediction is high enough, $e(n)$ tends to 0 . Then $c(n)-\hat{x}(n)$ and $s(n)$ tend to 0 recpectively. $e(n)$ and $s(n)$ have very nearly equal values.It is easily understood that the regular signal disturbs tracks of strange attractors. The weaker the regular signal is, the less the disturbance is ,and the higher the prediction precision is. Therefore, regular signal can be estimated when SNR is low by utilizing prediction error.

\section{Simulation and Results}

To test validity of the algorithm above, simulation examples are demonstrated. Two kinds of pulse signals are extracted from a typical chaotic series. The $x$ component of output from Lorenz model is applied as chaotic ground noise. Eliminating transiting points, a testing data of 2000 points is selected. The testing data are normalized as follow:

$$
x(n)=\frac{y(n)-\frac{1}{N} \sum_{k=1}^{N} y(k)}{\max (y(n))-\min (y(n))}
$$

where $\{y(n)\}$ is the original series, and $\{x(n)\}$ is the normalized time series. The first 1000 points are training samples, while the last $N_{p}=1000$ are testing samples. Mean square error of one-step prediction is defined as:

$$
\operatorname{Perr}=\frac{\sum_{n=1}^{N_{p}}[x(n)-\hat{x}(n)]^{2}}{\sum_{n=1}^{N_{p}} x^{2}(n)}
$$


Add a pulse signal $S(n)$ between the $1^{\text {st }}$ point to the $300^{\text {th }}$ point to the testing samples. To do quantitative analysis, Signal Chaos Ratio (SCR) is defined:

\section{Extraction of Sinusoidal Signal}

$$
S C R=10 \lg \left(\frac{P_{\text {signal }}}{P_{\text {chaos }}}\right)=10 \lg \left[\frac{\frac{1}{300} \sum_{n=1}^{300} S^{2}(n)}{\frac{1}{1000} \sum_{n=1001}^{2000} x^{2}(n)}\right](d B)
$$

The signal added here is a series of sinusoidal signals with normalized frequency 0.25 and amplitudes varying from -100dB to 0dB.Weak sinusoidal signals are extracted by applying Volterra filter. For the x component of Lorenz model, optimized parameters of phase space reconstruction of are set as follows: embedding dimension $\mathrm{m}=3$, and delay time $\tau=11$.

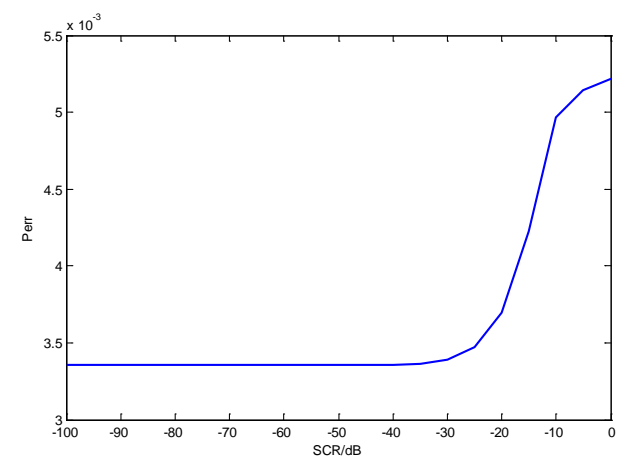

Fig.1 The prediction error of chaotic noise mixed with sinusoidal signal

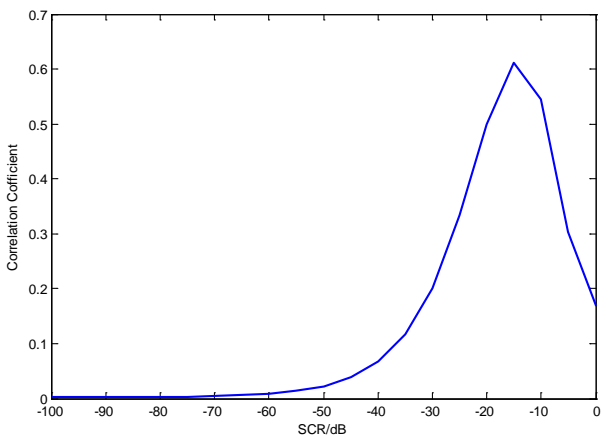

Fig. 2 The correlation coefficient of prediction error and sinusoidal signal

The extraction results of sinusoidal signals from Lorenz series are shown in Fig.1 and Fig.2.With increasing of SCR, the mean square error of one-step prediction remains stable until SCR reaches $-30 \mathrm{~dB}$. When $S C R \geq-30 \mathrm{~dB}$, the prediction error changes dramatically. It is because that the regular signals disturb tracks of strange attractors. The higher SCR is, the more significant the disturbance is. Solving cross-correlation of one step prediction error and the sinusoidal signal, similarity of waveforms are compared. When SCR is between $-30 \mathrm{~dB}$ to $0 \mathrm{~dB}$, waveform of prediction error is more close to that of sinusoidal signal. Thus sinusoidal signal can be extracted in this condition.

\section{Extraction of LFM Signal}

We also consider LFM signal mixed with the $x$ component of Lorenz model. The frequency band $\mathrm{B}=0.4 \mathrm{fs}$, and the amplitudes vary from $-100 \mathrm{~dB}$ to 0dB.As is shown in Fig.3 and Fig.4, weak LFM signals are extracted from chaotic background noise by using Volterra prediction model. Significantly, the cross correlation of prediction error and LFM signal appear lower. LFM series is broadband in frequency spectrum When the signals are mixed, the original strange attractor is disturbed from many frequency points, resulting in a larger prediction error. 


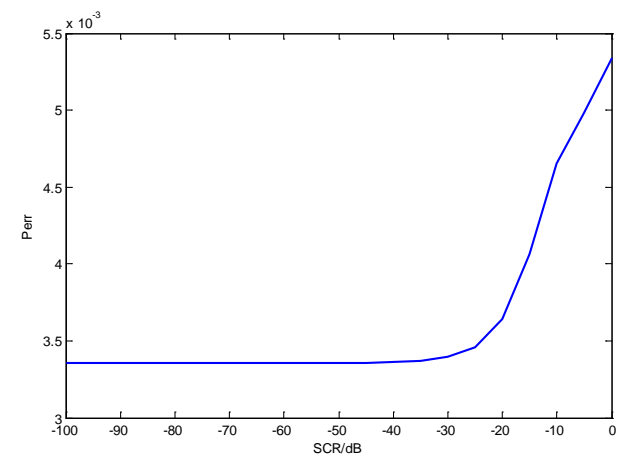

Fig.3 The prediction error of chaotic noise mixed with LFM signal

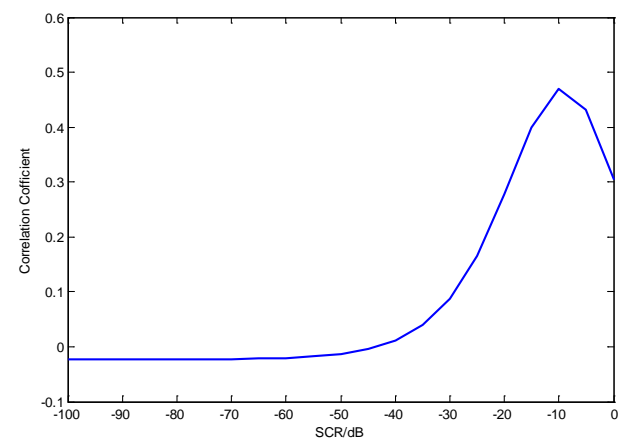

Fig.4 The correlation coefficient of prediction error and LFM signal

\section{Experimental results and analysis}

In practice, signals are always not regular. For example, in underwater acoustic target detection, the target is a certain ship-radiated noise, and interferences include ship-radiated noise, ambient noise, and so on. To verify the validity and robustness of the extraction method, one target signal is extracted from another ship-radiated by applying Volterra prediction model. All the ship-radiated noises were collected by standard acoustic sensors with an A/D conversion scheme of $10 \mathrm{kHz}$ sampling rate and a 16-bit resolution. Collected data was filtered by a low-pass filter whose upper limit is $1.6 \mathrm{kHz}$

\section{Modeling of Background noise}

One kind of merchant ship is marked Target1. In this thesis, the average mutual information (AMI) method is applied to estimate delay time. Embedding dimension is calculated by Cao method ${ }^{[10]}$. Optimized parameters of phase space reconstruction of are set as follows: embedding dimension $\mathrm{m}=6$, and delay time $\tau=14 T_{s}$.Utilizing Volterra model, one-step prediction results are obtained. As is indicated in Fig.5, prediction values and true values match well with each other. The mean square error Perr $=1.14 \times 10^{-3}$ can be calculated from one step prediction error.
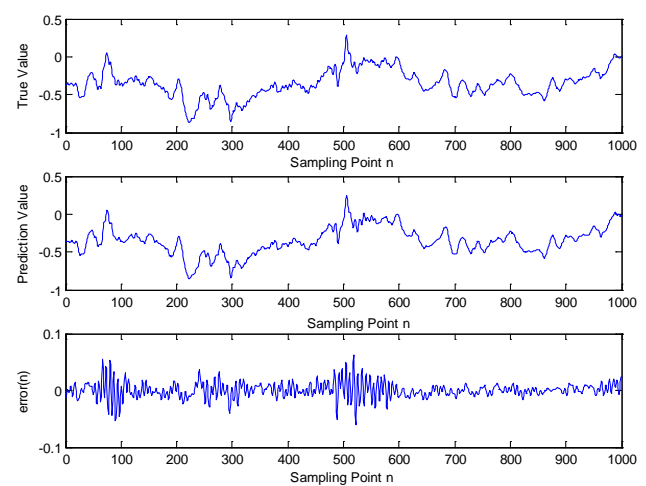

Fig.5 The prediction results of Target 1 


\section{Extraction Results and Analysis}

Target1 signal serves as background noise. Another kind of target signal with normalized amplitudes is added to the background noise between the $1001^{\text {th }}$ point to $1300^{\text {th }}$ point.SCR varies from $-30 \mathrm{~dB}$ to 30dB.Two kinds of other underwater acoustic target signals are marked Target 2 and Target 3,repectively. According to the nonlinear model in Section 3.1, one-step prediction results of mixed signals are gained. As is shown in Fig.6, the prediction error changes dramatically when $S C R \geq 0 d B$, indicating the appearance of the signal. Cross-correlation of one step prediction error and the Target 2 signal is illustrated in Fig.7. When SCR is larger than 10dB, waveform of prediction error is more close to that of Target 2 signal. To test the robustness of method, we also consider Target 3 signal mixed with Target 1 signal. The prediction results are shown in Fig.8 and Fig.9.

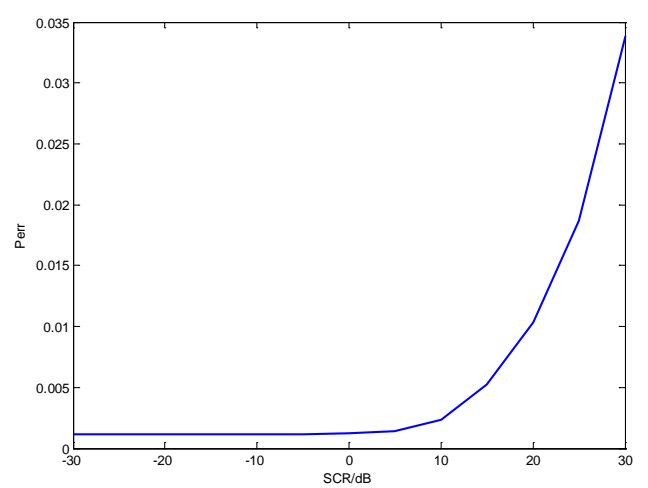

Fig.6 The prediction error of Target 1mixed with Target 2

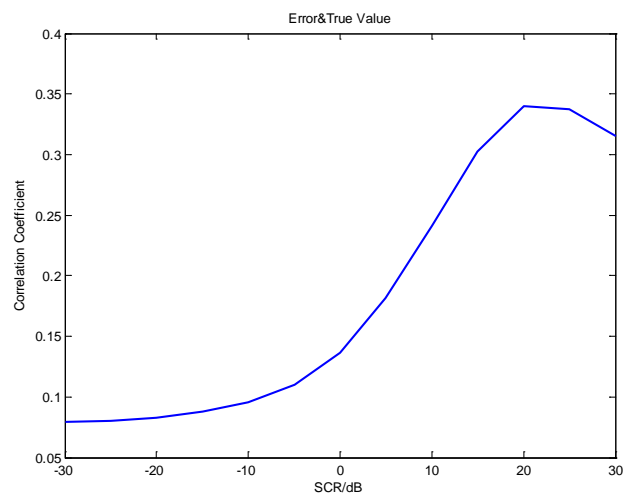

Fig.7 The correlation coefficient of prediction error and Target 2

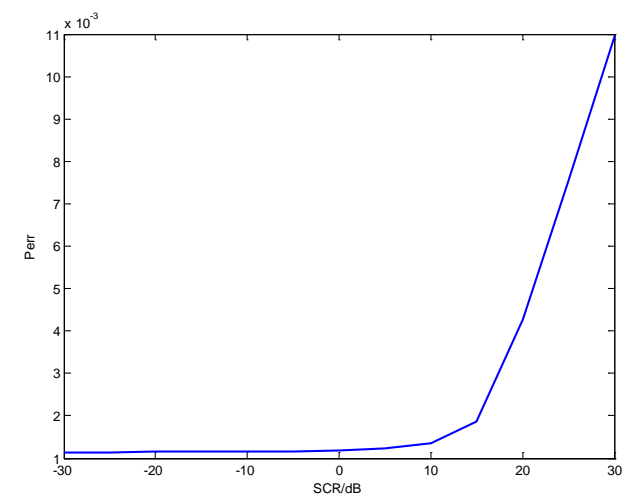

Fig.8 The prediction error of Target 1mixed with Target 3

\section{Summary}

In this paper, a method of extracting underwater acoustic weak signal base on Volterra filter is proposed. The method has a good prediction performance of chaotic noise. When regular signal is mixed with chaotic noise, the prediction error varies dramatically. As the model responds slowly to status changes of nonlinear system, signals can be extracted from strong chaotic noise. For verifying 
the validity of above method in detection of regular signals and ship noise, experimental data are used for computation. The method proves powerful for regular signal extraction, but for ship noise, only if SCR is higher than $10 \mathrm{~dB}$, the predicted waveform of ship noise can be acceptable.

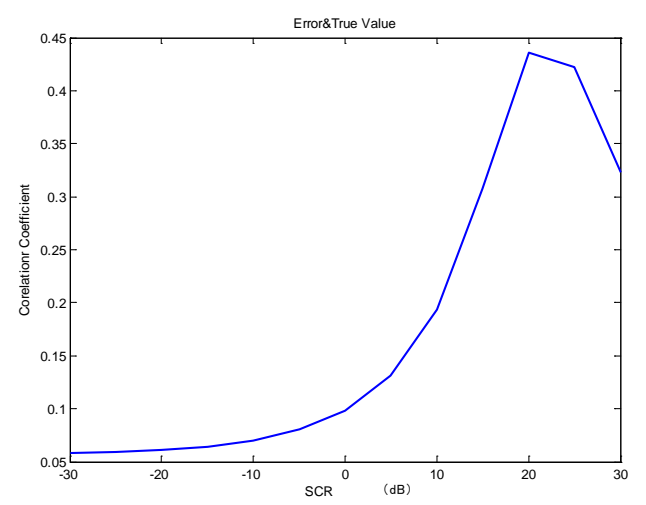

Fig.9 The correlation coefficient of prediction error and Target 3

\section{Acknowledgment}

This paper is supported by the key project of National Natural Science Fund (11234002).

\section{References}

[1] P.K. Hou, Y.F. Gong, X. Z. Shi and L. J. Lin: Detecting nonlinearity in the radiated noise of underwater targets. ACTA ACUSTICA. Vol. 26(2) (2001), p. 135-139

[2] X. H. Zhang, X. M. Zhang and L. J. Lin: Researches on Chaotic Phenomena of Noises Radiated from Ships. ACTA ACUSTICA. Vol.23(2) (1998),p. 134-140

[3] Frison T W et al: Chaos in Ocean Ambient “Noise”. J. Acoust. Soc. Am. Vol. 99(3) (1996), p.1527-1539

[4] Z. M. Cai, Z. N. Zheng and S. E. Yang, Chaos characteristic analysis of underwater reverberation. ACTA ACUSTICA. Vol. 27(6)(2002): 498-502

[5] J. Y. Pan, J. Han and S. E. Yang. A Neural Network based Method for Detection of Weak Underwater Signals. J. Marine Sci. Appl. Vol.( 9 )(2010): 256-261

[6] Z. M. Cai. The Study of Underwater Reverberation Modeling and Processing Based on Dynamical System. (Harbin Engineering University, Harbin 2002)

[7] D. M. Hummels, W. Ahmed, M.T. Musavi. Adaptive detection of small sinsusoidal signals in non-Gaussian noise using an RBF neural network. IEEE Transactions on Neural Networks. Vol.6(1) (1995),p. 214-219.

[8] V. J. Mathews. Adaptive polynomial filters. Signal Processing Magazine, IEEE, 8(3) (1991), p.10-26

[9] V.N.Vapnik. The nature of statistical learning theory (Springer-Verlag, New York 1999)

[10] J. H. LV. Analysis and application of chaotic time series (Wuhan University Press, Wuhan 2005) 